A Si ngl e- Pol ar i zat i on Hol ey Fi ber Wth Ani sotropi c Lattice of Circul ar Air Hol es

\begin{tabular}{|l|l|}
\hline 著者 & $\begin{array}{l}\text { I CH KAMA Kazuki, ZHANG Zej un, TSUJI } \\
\text { Yasuhi de, EGUCH } \\
\text { Nasashi }\end{array}$ \\
\hline $\begin{array}{l}\text { j our nal or } \\
\text { publ i cat i on ti t l e }\end{array}$ & $\begin{array}{l}\text { Journal of I i ght wave t echnol ogy : a j oi nt } \\
\text { I EEE/OSA publ i cat i on }\end{array}$ \\
\hline vol une & 33 \\
\hline number & 18 \\
\hline page r ange & $3866-3871$ \\
\hline year & 2015- 09-15 \\
\hline URL & ht t p: //hdl . handl e. net /10258/3827 \\
\hline
\end{tabular}


A Si ngl e- Pol ar i zat i on Hol ey Fi ber Wth Ani sotropi c Lattice of Circul ar Air Hol es

\begin{tabular}{|l|l|}
\hline 著者 & $\begin{array}{l}\text { I CH KAMA Kazuki, ZHANG Zej un, TSUJI } \\
\text { Yasuhi de, EGUCH } \\
\text { Nasashi }\end{array}$ \\
\hline $\begin{array}{l}\text { j our nal or } \\
\text { publ i cat i on ti t l e }\end{array}$ & $\begin{array}{l}\text { Journal of I i ght wave t echnol ogy : a j oi nt } \\
\text { I EEE/OSA publ i cat i on }\end{array}$ \\
\hline vol une & 33 \\
\hline number & 18 \\
\hline page r ange & $3866-3871$ \\
\hline year & 2015- 09-15 \\
\hline URL & ht t p: //hdl . handl e. net /10258/3827 \\
\hline
\end{tabular}




\title{
A Single-Polarization Holey Fiber with Anisotropic Lattice of Circular Air Holes
}

\author{
Kazuki ICHIKAWA, Student member, IEEE, Zejun ZHANG, Student member, IEEE, Yasuhide TSUJI, Member, \\ IEEE, Member, OSA, and Masashi EGUCHI, Member, IEEE, Senior Member, OSA,
}

\begin{abstract}
We study on a novel single-polarization single-mode (SPSM) holey fiber (HF) using an anisotropic lattice of air holes. Elliptical hole core circular hole HFs (EC-CHFs) recently proposed can easily realize single-polarization regime by utilizing the anisotropic fundamental space-filling modes (FSMs) of the air hole lattices. In this paper, in order to realize an SPSMHFs based on the same principle of the SPSM regime in the EC-CHF without using elliptical air holes, we use an anisotropic arrangement of circular air holes in the core region. In order to obtain Gaussian like mode profile, the dependence of the FSM and mode profile on the air hole sizes, lattice constant, and air hole arrangement in the core region are investigated in detail.
\end{abstract}

Index Terms-single-polarization transmission, photonic crystal fiber, anisotropic lattice, birefringence

\section{INTRODUCTION}

Over the past few decades, the Internet traffic is rapidly growing, so that it is expected that conventional optical fibers will become inadequate. Under this circumstance, in order to expand the communication capacity, various new types of high performance optical fibers have been extensively studied. One of these fibers is photonic crystal fibers (PCFs) with interesting features such as endlessly single mode (ESM) behaver, high nonlinearity and so on [1]- [4]. The PCFs can be generally classfied into two types according to their guidance mechanism. One is holey fiber (HF) [4], in which light is guided by total internal reflection, and the other is photonic bandgap fiber (PBGF) [4], in which light is guided by photonic bandgap effect.

In the case of ordinary HFs with a triangular lattice of air holes, the structure has a sixfold symmetry and the two orthogonally polarized modes in these fibers are degenerate. In order to realize birefringent or single polarization fibers, some asymmetries have to be introduced in the core and/or cladding region, and several kinds of structures have been reported so far [5]- [12]. The elliptical-hole core circular-hole HF (EC-CHF), which is one of the novel single-polarization HFs, can be easily achieved by using the effective index difference between the fundamental space-filling mode (FSM) of circular-hole lattice in the cladding and the anisotropic FSM of elliptical-hole lattice in the core region [13]- [17]. In this paper, we present a novel single-polarization and singlemode (SPSM) HF with circular air-holes in the core. In this structure, the SPSM regime can be easily achieved by utilizing

K.Ichikawa, Z. Zhang and Y. Tsuji are with the Division of Information and Electronic Engineering, Muroran Institute of Technology, Muroran, 050-8585 Japan. M. Eguchi is with the Department of Photonics System Technology, Chitose Institute of Science and Technology, Chitose 066-8655, Japan.

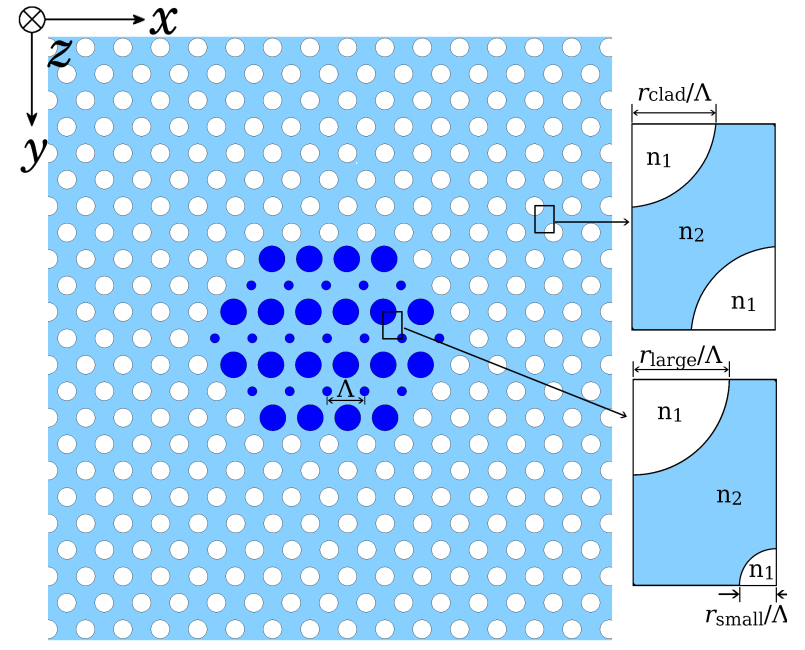

Fig. 1. Single-polarization HF with anisotropic lattice of large and small air-holes in the core.

the anisotropic FSM of the core lattice, in the same guidance mechanism as the EC-CHF. Similar approach to introduce the birefringence in core is reported in Ref. [12]. However, in Ref. [12], high index rods with a smaller lattice pitch compared to the cladding are used in core region and SPSM-HF is not demonstrated with this structure. Our SPSM-HF consists of only air holes and the lattice pitch is same in the core and cladding.

In Section II, we investigate the SPSM regimes of our proposed HF which has circular air holes in the core, whose sizes periodically change in the specific direction. Finally, we conclude in Section III.

\section{SPSM-HF WITH ANISOTROPIC LATTICE OF CIRCULAR AIR HOLES.}

In this section, we investigate a novel structure of SPSM-HF only with circular air-holes although it is based on the same principle of EC-CHF, which has large birefringence in the core region utilizing anisotropic FSM of the elliptical air hole lattice. On the other hand, the present HF utilize anisotropic arrangement of circular air holes.

\section{A. HF with large and small air-holes in the core region.}

We investigate the novel structure of HF with a core lattice consisting of inhomogeneous air holes. The cross-section of the HF and the analysis regions of each FSM are shown in 


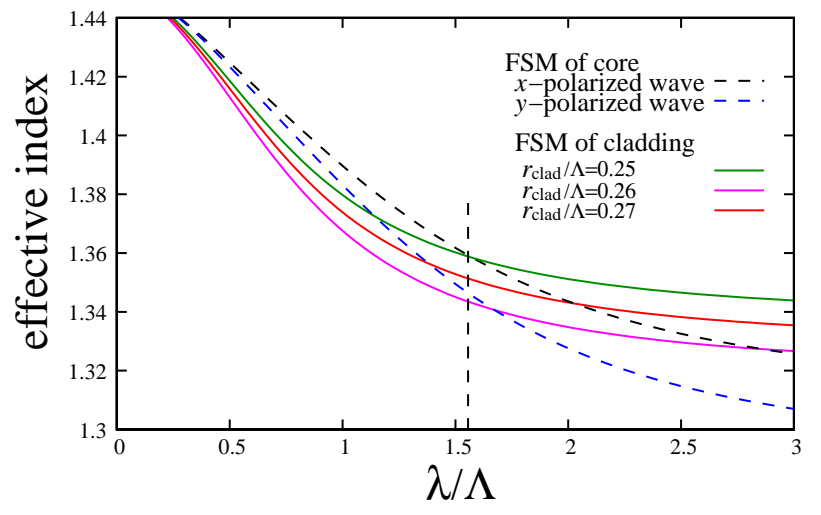

Fig. 2. Effective indices of the core and cladding FSM of the HF shown in Fig. 1. The thick dashed curve show FSM of the core (black and blue curves mean $x$ - and $y$-polarized wave, respectively) and the thick solid curves show FSM of the cladding from the top to bottom, inorder, $r_{\text {clad }} / \Lambda=0.25,0.26$, and 0.27 .

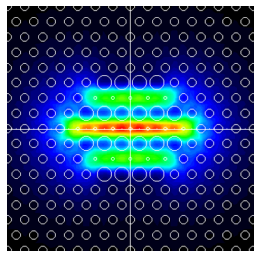

(a) $E_{11}^{x}$ mode

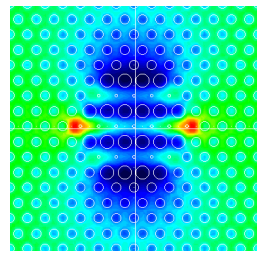

(b) $E_{11}^{y}$ mode

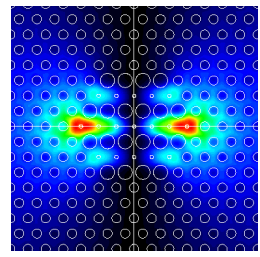

(c) $E_{21}^{x}$ mode
Fig. 3. Field distribution at $\lambda / \Lambda=1.55$ for (a) $E_{11}^{x}$ mode, (b) $E_{11}^{y}$ mode, and (c) $E_{21}^{x}$ mode of the HF as shown in Fig. 1.

Fig. 1. The structure has an asymmetry in the core whose air hole sizes are modified periodically along the $y$-direction. In this paper, the large and small air-hole radii in the core region are referred to $r_{\text {large }}$ and $r_{\text {small }}$, respectively, and the air-hole radius in the cladding region is referred to $r_{\text {clad }}$. The lattice pitch is $\Lambda$, and the hole radii are set to be $r_{\text {large }} / \Lambda=0.4$ and $r_{\text {small }} / \Lambda=0.1$, respectively. The light blue region indicates $\mathrm{SiO}_{2}$, and the blue or white regions indicate air holes. The holes indicated by blue are the air holes which constitute the anisotropic core.

In order to design an SPSM-HF, first we investigated the birefringence of the FSM of the anisotropic core lattice. The dispersion curves of the FSMs for the core and cladding regions are shown in Fig. 2. The dispersion curves for the cladding lattice are shown for $r_{\text {clad }} / \Lambda=0.25,0.26$, and 0.27 , respectively. The lattice with smaller air holes has a higher effective index. For example, in the case of $\Lambda=1 \mu \mathrm{m}$, in order to achieve single-polarization operation in the conventional communication band $\lambda / \Lambda=1.55$, the $\mathrm{HF}$ with $r_{\text {clad }} / \Lambda=0.26$ can be designed.

Figure 3 shows the field distribution of the eigen modes of our proposed HF. The vector finite element method with higher-order curvilinear edge and nodal hybrid elements is employed to obtain these eigen modes [18]. Only the $x$ polarized wave is confined in the core region and the $y$ polarized wave is a radiation mode. We can see that the singlepolarized operation is achieved according to these result.

However, the fundamental modal field distribution of this fiber does not match with those of conventional fiber modes.

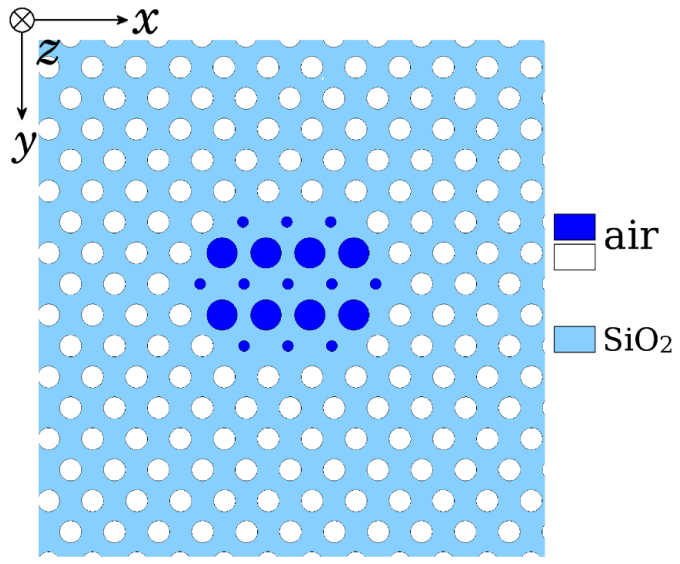

Fig. 4. 2-ring core HF consist of the large and small air-holes in the core.

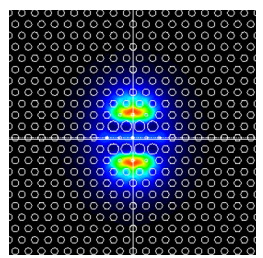

(a) $E_{11}^{x}$ mode

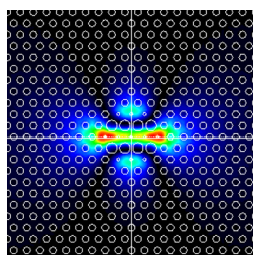

(b) $E_{11}^{y}$ mode

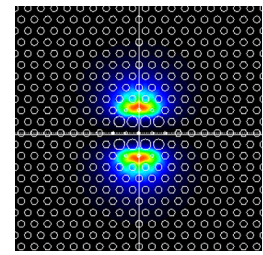

(c) $E_{12}^{x}$ mode
Fig. 5. Field distribution at $\lambda / \Lambda=1.55$ for (a) $E_{11}^{x}$ mode, (b) $E_{11}^{y}$ mode, and (c) $E_{21}^{x}$ mode of the HF as shown in Fig. 4 .

The field distribution has a lot of relatively large dips, especially along the $y$-direction. Additionally, from Fig. 3 (c), the higher order mode is also supported in this fiber. Therefore, in order to achieve the single-mode operation, based on the conventional optical fiber theorem, we consider the maximum core radius, $R_{\mathrm{core}}^{\max }$, as follows,

$$
R_{\text {core }}^{\max }=\frac{V_{c} \lambda}{2 \pi n_{1} \sqrt{2 \Delta}}
$$

where $\Delta$ is a relative refractive index difference which is given as

$$
\Delta=\frac{n_{1}^{2}-n_{2}^{2}}{2 n_{1}^{2}} .
$$

Here, $n_{1}$ and $n_{2}$ are the effective indices of the FSMs in the core and cladding regions, respectively, $V_{c}$ is the cutoff (normalized frequency, $V$-value), and $\lambda$ is the operating wavelength. $V$-value is required to be smaller than $V_{c}=2.4048$ to realize the single-mode operation. From (1), the core radius $R_{\text {core }}$ has to be smaller than $2.5 \Lambda$ to realize the single-mode regime at the considered wavelength.

Next, in order to realize the SPSM regime, we investigate a 2-ring structure of $\mathrm{HF}$ as shown in Fig. 4. The other parameters are the same as the previous structure. Figure 5 shows the field distribution of the eigen modes in the $\mathrm{HF}$ shown in Fig. 4. Unexpectedly, we can observe that both the $x$ - and $y$-polarized waves are supported and the $x$-polarized higher-order mode is also supported. The effective indices 


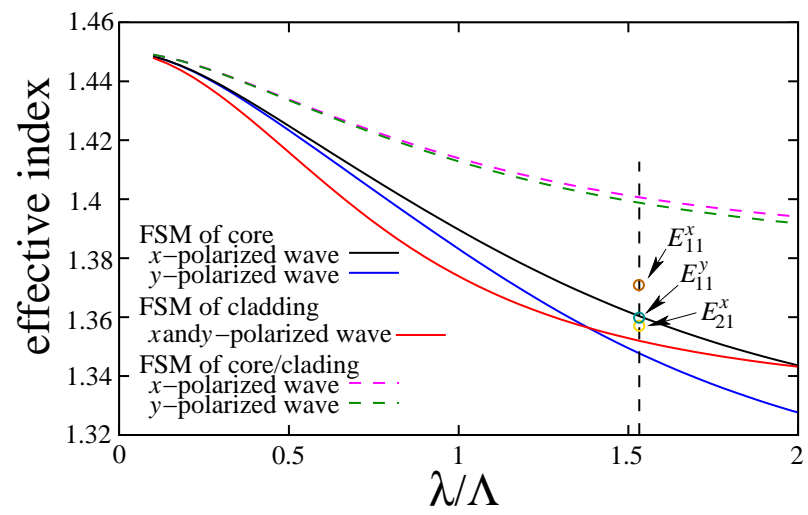

Fig. 6. Effective indices of the FSM in core, cladding, and core-cladding boundary of the HF shown in Fig. 4. The black and blue thick solid curves show the effective indices of FSMs of the core for $x$ - and $y$-polarized wave, respectively, and the red thick solid curve shows the cladding FSM. The magenta and green dashed curves show the effective indices of the corecladding boudary FSMs of $x$ - and $y$-polarized wave, respectively.

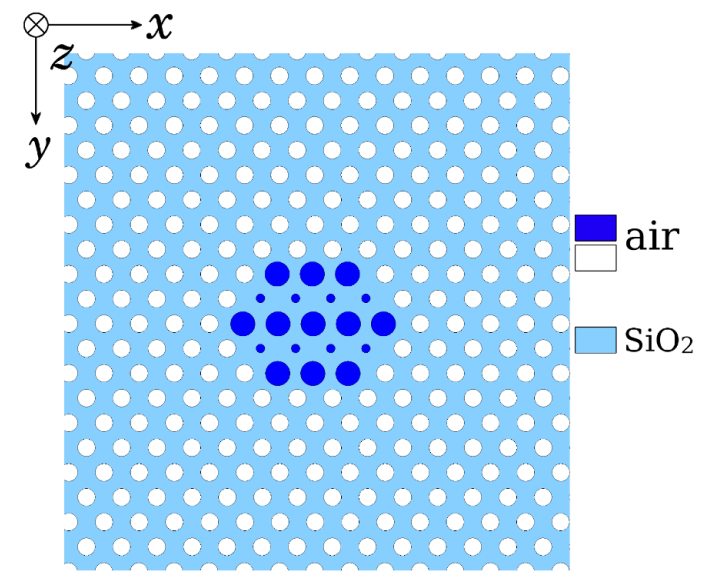

Fig. 7. 2-ring SPSM-HF with inverted position of the large and small air holes in the core.

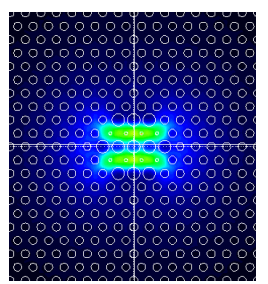

(a) $E_{11}^{x}$ mode

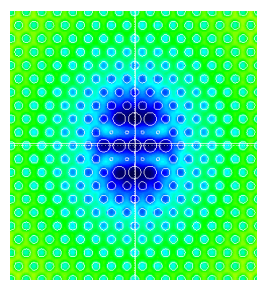

(b) $E_{11}^{y}$ mode

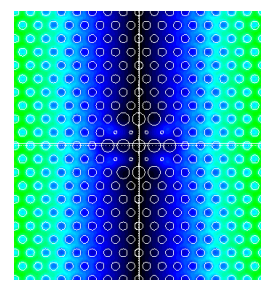

(c) $E_{21}^{x}$ mode
Fig. 8. The field distribution at $\lambda / \Lambda=1.55$ for (a) $E_{11}^{x}$ mode, (b) $E_{11}^{y}$ mode and (c) $E_{21}^{x}$ mode of the HF as shown in Fig. 7.

of $E_{11}^{x}, E_{11}^{y}$, and $E_{21}^{x}$ modes are 1.364, 1.359, and 1.358, respectively. The dispersion curves of the core, cladding, and core-cladding boundary regions are respectively shown in Fig. 6 . The effective indices of the propagating modes shown in Fig. 5 are higher than those of the FSMs in the core and cladding regions. In these eigen modes, the fields are localized around the core-cladding boundary, rather than in the core region. In this HF, the outermost air holes of the core are the smaller ones and the effective index of the core-cladding boundary lattice is higher than those of the core or cladding lattice. This is the reason why we cannot realize SPSM in

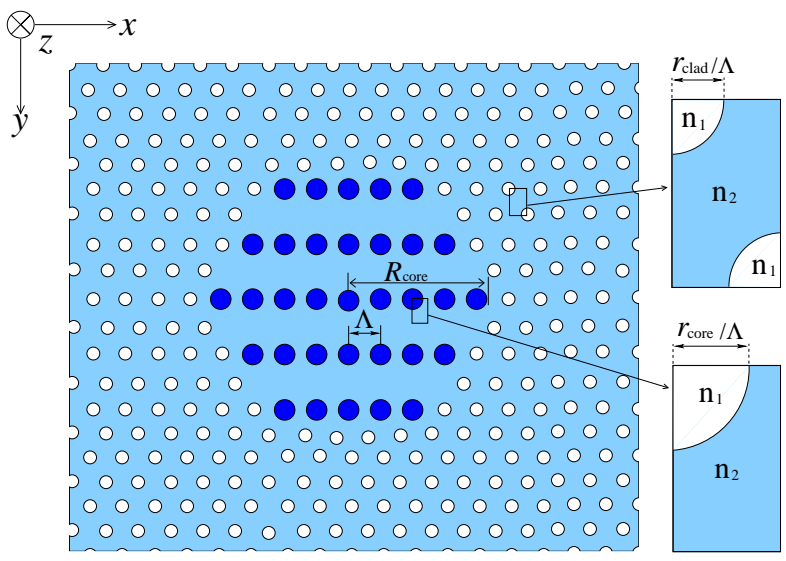

Fig. 9. 4-ring SPSM-HF consisted of only large hole at $\lambda / \Lambda=3.0$.

the structure shown in Fig. 4. In order to confirm this, we estimated the effective index of FSM for the core-cladding boundary lattice. The results are shown in Fig. 6. The effective indices of the $x$ - and $y$-polarized fundamental modes are also plotted. We can see that the effective indices of three modes of the HF exist between those of the boundary and cladding modes.

In order to suppress the boundary modes of the corecladding region, we consider an $\mathrm{HF}$ as shown in Fig. 7. In this structure, the large and small air holes are rearranged and the outermost air holes of the core are the large air holes. Figure 8 shows the eigen field distribution of the HF shown in Fig. 7 . We can see that only the $x$-polarized fundamental mode is supported and the SPSM operation is achieved. However, the mode field profile of this HF is significantly different from those of the standard single-mode fibers (SMFs) and the splice loss between an SMF and our proposed SPSM-HF is an important matter to be considered. The large dent in this HF is caused by the large air hole size compared to the wavelength. Therefore, next we design the SPSM-HF at longer normalized wavelengths.

\section{B. HF with only large air-hole in the core region}

The HF as shown in the previous subsection realizes the SPSM operation, but the mode field profile of this HF is significantly different from those of standard SMFs. In addition, we introduce small air-holes between the large air-holes to get a similar mode field profile to those of standard SMFs by reducing the refractive index difference within the core region. However, this approach is not quite effective to improve the mode field profile. In the following discussion, considering the practical fabrication, we eliminate the small air holes and, in the following discussion, $r_{\text {large }}$ is referred to as $r_{\text {core }}$.

1) the case of $\lambda / \Lambda=3$ : We consider a structure without small air-holes as shown in Fig. 9. The structural parameters are the same as those in the previous subsection except for the small air-hole size. Figure 10 shows the dispersion characteristics of the core and cladding FSMs, respectively. The dispersion curves of the cladding lattice are shown for $r_{\text {clad }} / \Lambda=0.26,0.27$, and 0.28 , respectively. In this design, the normalized wavelength is set to be $\lambda / \Lambda=3$ to improve 


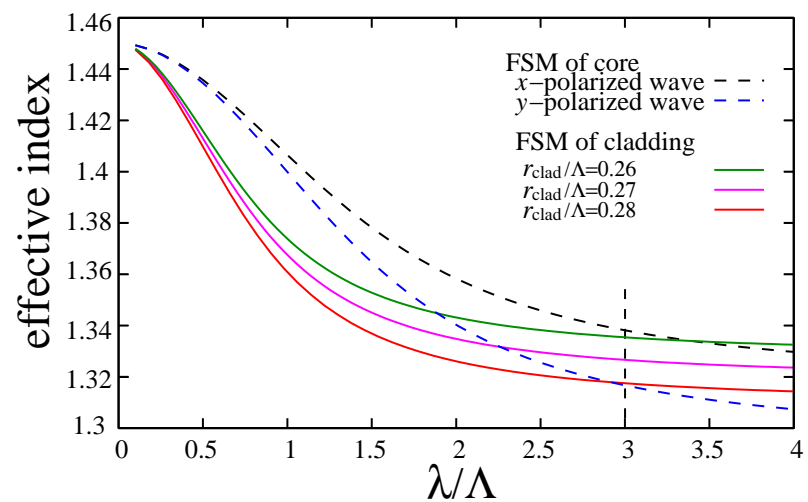

Fig. 10. Effective index of the core and cladding FSM. The thick dashed curve shows FSM of the core (the black and blue curve means $x$ - and $y$ polarized wave, respectively) and the thick solid curve shows FSM of the cladding (each curve means the air hole size $r_{\text {clad }} / \Lambda=0.26,0.27,0.28$, in order from the top).

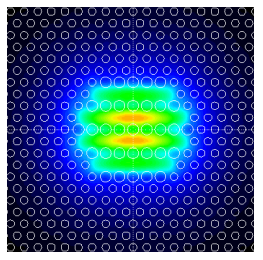

(a) $E_{11}^{x}$ mode

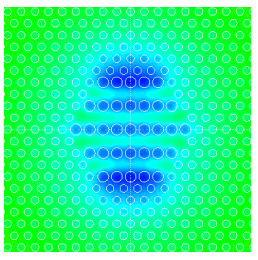

(b) $E_{11}^{y}$ mode

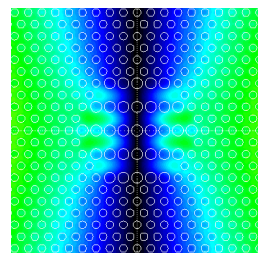

(c) $E_{21}^{x}$ mode
Fig. 11. The field distribution at $\lambda / \Lambda=3$ for (a) $E_{11}^{x}$ mode, (b) $E_{11}^{y}$ mode and (c) $E_{21}^{x}$ mode of the HF as shown in Fig. 9.

the mode field profile. This HF is able to be used in the communication band by setting the lattice constant $\Lambda=0.5 \mu \mathrm{m}$. From Fig. 10, the SPSM operation is able to be realized for both $r_{\text {clad }} / \Lambda=0.26,0.27$ and 0.28 . Here, in order to get a stronger light confinement, we employ $r_{\text {clad }} / \Lambda=0.28$. In this case, the core-radius $R_{\text {core }}$ for the single mode operation has to be $R_{\text {core }} \lesssim 4.8 \Lambda$ and the 4-ring SPSM-HF is investigated.

In this design, in order to suppress unwanted surface modes, the air holes in the core are arranged so that the large air holes are located at the outermost core ring. Figure 11 shows the field distribution of the HF. We can see that only the $x$-polarized fundamental mode is supported and the SPSM regime is achieved. The mode profile of this HF also has a slight irregularity.

2) the case of $\lambda / \Lambda=4.5$ : In order to obtain a Gaussianlike mode field profile, we consider further longer normalized wavelengths (smaller lattice constant) and set $\lambda / \Lambda$ to be 4.5 . The structural parameters are the same as those used in the previous discussion except for the lattice constant and the core geometry. The core radius is assumed to be $R_{\text {core }} \lesssim 7.8 \Lambda$ and we consider a 6-ring SPSM-HF shown in Fig. 12. The large air holes, which constitute the core, are arranged so that the core geometry is almost circular.

The dispersion properties of the core and cladding FSMs for both $x$ - and $y$-polarized waves are shown in Fig. 13. From this figure, we can see that the SPSM operation is able to be realized for $r_{\text {clad }} / \Lambda=0.265 \sim 0.289$. In the following structure, in order to get a stronger light confinement, we employ $r_{\text {clad }} / \Lambda=0.285$. Figure 14 shows the field distribution

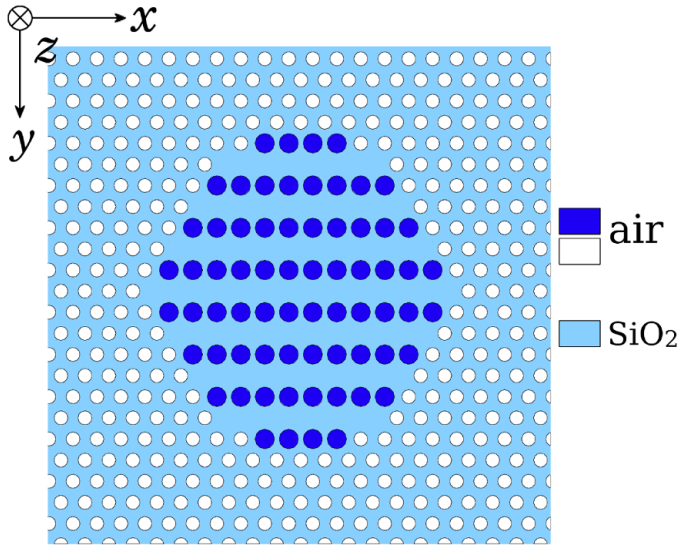

Fig. 12. 6-ring SPSM-HF designed at $\lambda / \Lambda=4.5$.

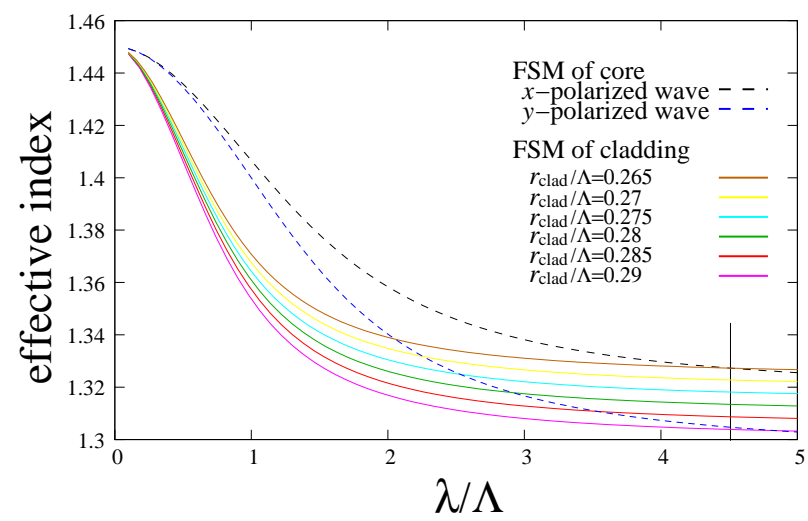

Fig. 13. Effective indices of the core and cladding FSM. The thick dashed curves show FSM of the core (each curve means $x$ - and $y$-polarized wave, in order from the top) and the thick solid curves show FSM of the cladding (each curve means the air hole size $r_{\text {clad }} / \Lambda=0.265,0.27,0.275,0.28$, 0.285 , and 0.29 in order from the top).

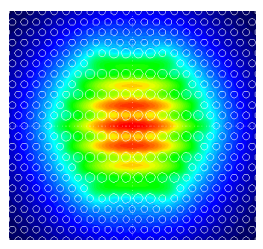

(a) $E_{11}^{x}$ mode

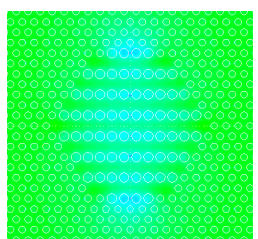

(b) $E_{11}^{y}$ mode

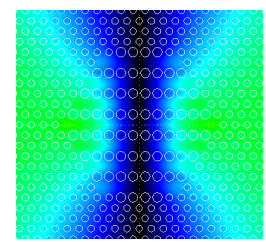

(c) $E_{21}^{x}$ mode
Fig. 14. Field distribution at $\lambda / \Lambda=4.5$ for (a) $E_{11}^{x}$ mode, (b) $E_{11}^{y}$ mode and (c) $E_{21}^{x}$ mode of the HF as shown in Fig. 12.

of the eigen modes of this HF. In this HF, only $E_{11}^{x}$ mode is supported and the SPSM operation is realized. Moreover, comparing with the previous HF, the modal field profile is improved to match a Gaussian profile. This is because, in longer wavelength range, the optical field is less confined in the higher refractive index region and the more flat field distribution can be obtained. In the next subsection, in order to make it clear, we investigate the relationship between the modal field and waveguide parameters.

Next, in order to evaluate the mode matching to the Gaussian field, we calculate the overlap integrals between the mode field of SPSM-HF and a Gaussian field. Figure 15 shows the 


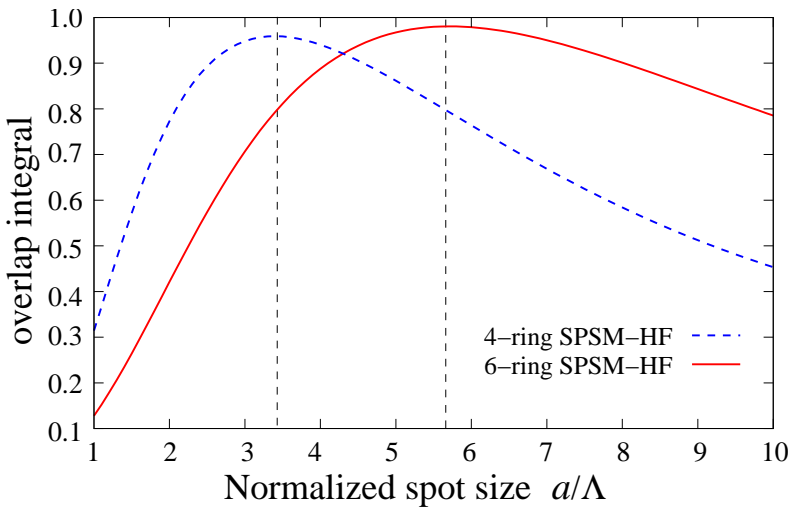

Fig. 15. The result calcurating the overlap integral with $\mathrm{HE}_{11}^{x}$ of SPSM-HF and Gaussian profile.

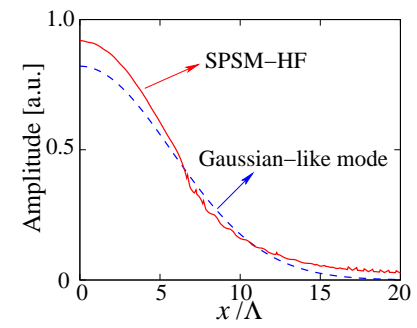

(a)

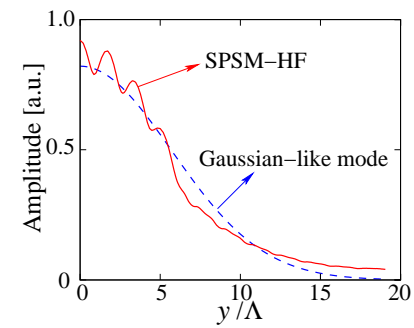

(b)
Fig. 16. The mode field distribution of SPSM-HF shown in Fig. 14 (a) and a Gaussian profile. Field distribution along the (a) $x$-axis and (b) $y$-axis with the origin at the center of the core.

overlap integrals as a function of the normalized spot size of a Gaussian profile, $a / \Lambda$. We note that the maximum overlap for 4-ring SPSM-HF shown in Fig. 9 can be up to $96 \%$ at the normalized spot size $a / \Lambda=3.4$. Furthermore, the mode matching to the Gaussian field is improved up to $98 \%$ at $a / \Lambda=5.7$ in the case of 6-ring SPSM-HF shown in Fig. 12 . Figures 16 (a) and (b) show the mode field distributions of 6ring SPSM-HF along the $x$ - and $y$-axis with the origin at the center of the core, respectively. The solid red and dashed blue curves represent the mode field distributions of the SPSM-HF and a Gaussian profile, respectively. From Figs. 16 (a) and (b), we can see that each mode profile has fairly good mode matching to the Gaussian profile.

3) The modal field and waveguide parameters: Figure 17 shows the magnetic field distribution of the FSM for the several waveguide parameters. As expected, it can be seen that the flatness of the field is higher for the smaller lattice constant and smaller air holes. Fig. 18 shows the relationship between the air hole size and the birefringence of the core lattice. In Fig. 18, the maximum core-radius for the single mode operation is also shown. Although the core lattices with $\left(r_{\text {core }} / \Lambda, \lambda / \Lambda\right)=(0.2,1.5)$ and $(0.4,3.0)$ have almost same degree of flatness, the birefringence is much higher for $\left(r_{\text {core }} / \Lambda, \lambda / \Lambda\right)=(0.4,3.0)$. From, these results, the birefringence of FSM are plotted as a contour map for $\lambda / \Lambda$ and $r_{\text {core }} / \Lambda$ in Fig. 19. From this figure, for longer normalized wavelengths $\lambda / \Lambda>3$, the birefringence is almost determined only by the air filling fraction (the normalized air hole radius $r_{\text {core }} / \Lambda$ ).

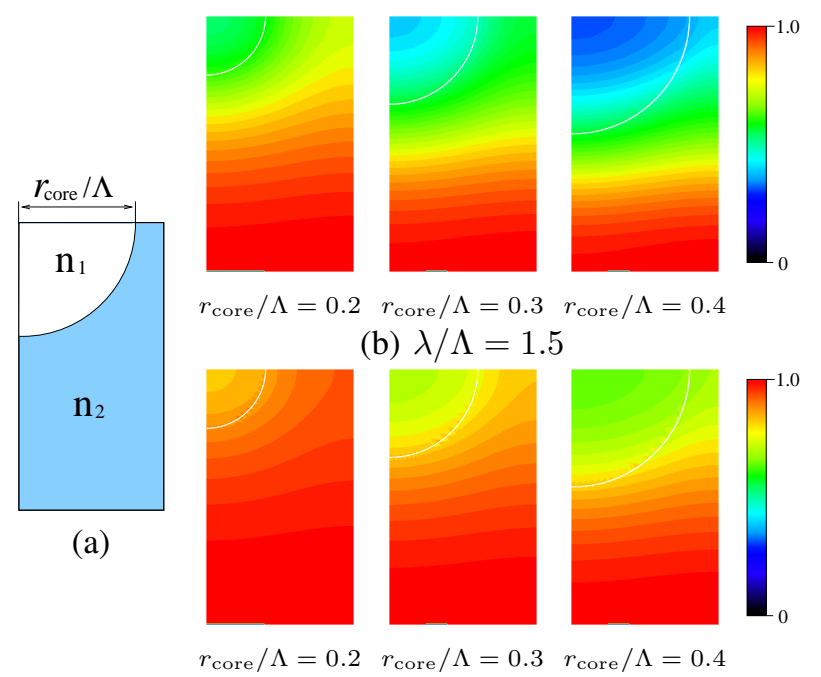

(c) $\lambda / \Lambda=3.0$

Fig. 17. The magnetic field distribution of the core FSM with different air hole sizes and lattice pitches, (a) Analysis region of the FSM in core, (b) Field distributions with different core sizes at $\lambda / \Lambda=1.5$, (c) Field distributions with different core sizes at $\lambda / \Lambda=3.0$.

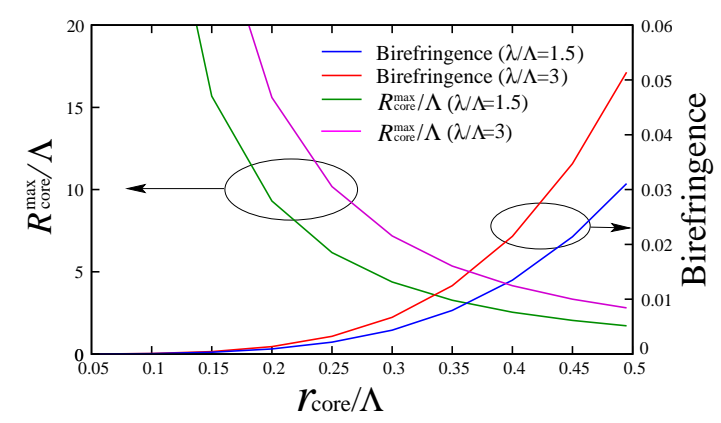

Fig. 18. The relations among hole size in the core, birefringence, and $R_{\text {core }}$. the blue and red solid curve show birefringence for $\lambda / \Lambda=1.5$ and $\lambda / \Lambda=$ 3.0 , respectively. the green and magenta solid curves show $R_{\text {core }}$ for $\lambda / \Lambda=$ 1.5 and $\lambda / \Lambda=3.0$, respectively.

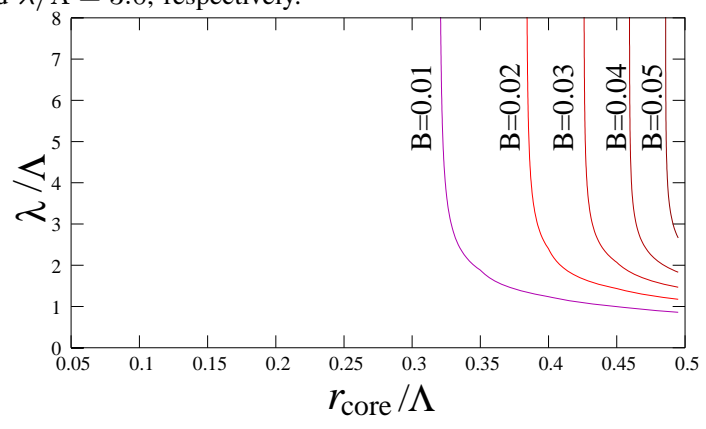

Fig. 19. The dependence of wavelength and air hole-size in the core, respectively, on the birefringence.

4) The influence of scattering loss: Since our proposed SPSM-HF has a lot of air holes in the core region, the scattering loss due to the air/silica surface roughness is concerned. In order to estimate the scattering effect, we investigate the factor $F$ which is usually used for the hollow core photonic band gap fibers (HC-PBGF) [19]. The estimated $F$ value is $F=2.61 \mu \mathrm{m}^{-1}$ for 6-ring SPSM-HF at the operating wavelength $\lambda=1.55 \mu \mathrm{m}(\Lambda=\lambda / 4.5 \simeq 0.334 \mu \mathrm{m})$. The $F$ 
value and the corresponding scattering loss reported in [20] are $0.0174 \mu \mathrm{m}^{-1}$ and $3.5 \mathrm{~dB} / \mathrm{km}$, respectively. Comparing to [20], the estimated $F$ value of our SPSM-HF is about 150 times larger and the corresponding scattering loss may be estimated at $525 \mathrm{~dB} / \mathrm{km}$. Therefore, our SPSM-HF does not seem to be favorable to long distance transmission. However, this scattering loss does not seem to be significant drawback for some applications such as a short length polarization splitting device proposed in Ref. [16], whose device length is around $1 \mathrm{~mm}$. In such application, the scattering loss of our proposed SPSM-HF can be negligible since the loss estimation is about $5.25 \times 10^{-4} \mathrm{~dB} / \mathrm{mm}$.

\section{CONCLUSION}

In this paper, we proposed a novel single-polarization HF and have investigated the structural parameters to achieve the SPSM-HF. In our structures, the birefringence of the core is introduced by the anisotropic arrangement of circular air-holes, and the single-polarization regime can be easily achieved by designing the hole size of the cladding. We also demonstrated that the modal field profile of the proposed HF is improved to match the Gaussian mode profile by reducing the lattice pitch. Considering the current fabrication technology and practical application, the performance of our proposed SPSM-HF may be degraded. Therefore in the future work, we will investigate the other important properties of our proposed SPSM-HF, such as the structural tolerance and the wavelength bandwidths for SPSM-HF.

\section{REFERENCES}

[1] J. C. Knight, T. A. Birks, P. St. J. Russell, and D. M. Atkin, "All-silica single-mode optical fiber with photonic crystal cladding," Opt. Lett., Vol. 21, No. 19, pp. 1547-1549, Oct. 1996.

[2] A. R. Bhagwat and A. L. Gaeta, "Nonlinear optics in hollow-core photonic bandgap fibers," Opt. Lett., Vol. 16, No. 7, pp. 5035-5047, Mar. 2008.

[3] T. A. Birks, J. C. Knight, and P. St. J. Russell, "Endlessly single-mode photonic crystal fiber," Opt. Lett., Vol. 22, No. 13, pp. 961-963, July 1997.

[4] J. Broeng, D. Mogilevstev, E. Barkou, and A. Bjarklev, "Photonic crystal fibers: a new class of optical waveguides," Opt. Fiber. Lett., Vol. 5, pp. 305-330, 1999.

[5] K. Saitoh and M. Koshiba, "Single-polarization single-mode photonic crystal fibers," IEEE Photon. Technol. Lett., Vol. 15, No. 10, pp. 13841386, Oct. 2003.

[6] H. Kubota, S. Kawanishi, S. Koyanagi, M. Tanaka, and S. Yamaguchi, "Absolutely single polarization photonic crystal fiber," IEEE Photon. Technol. Lett., Vol. 16, No. 1, pp. 182-184, Jan. 2004.

[7] J. R. Folkenberg, M. D. Nielsen, and C. Jakobsen, "Broadband singlepolarization photonic crystal fiber," Opt. Lett., Vol. 30, No. 12, pp. 14461448, June 2005.

[8] M. J. Steel and R. M. Osgood, "Polarization and dispersive properties of elliptical-hole photonic crystal fibers," J. Lightw. Technol., Vol. 19, No. 4, pp. 495-503, Apr. 2001.

[9] J. Ju, W. Jin, and M. S. Demokan, "Design of single-polarization singlemode photonic crystal fiber at 1.30 and $1.55 \mu \mathrm{m}$," J. Lightw. Technol., Vol. 24, No. 2, pp. 825-830, Feb. 2006.

[10] M. Szpulaka, T. Martynkiena, J. Olszewskia, W. Urbanczyka, T. Nasilowskib, F. Berghmansb, and H. Thienpont, "Single-polarization single-mode photonic band gap fiber," ACTA Phy. Pol. A, Vol. 111, No. 2, pp. 239-245, 2007

[11] G. Ryouichiro, J. D. Stuart, and T. Katsuhiro, "Single-polarization operation in birefringent all-solid hybrid microstructured fiber with additional stress applying parts," Opt. Lett., Vol. 34, No. 20, pp. 31193121, Oct. 2009.

[12] J. Broeng, P. Skovgaard, E. Knudsen, J. Jensen, M. Nielsen. "Optical waveguide, method of its production, and its use," U.S.Patent 0120677 A1, June 8, 2006

[13] M. Eguchi and Y. Tsuji, "Single-mode single-polarization holey fiber using anisotropic fundamental space-filling mode," Opt. Lett., Vol. 32, No. 15, pp. 2112-2114, Aug. 2007.

[14] M. Eguchi and Y. Tsuji, "Influence of reflected radiation waves caused by large mode field and large refractive index mismatches on splice loss evaluation between elliptical-hole lattice core holey fibers and conventional fibers," J. Opt. Soc. Am. B, Vol. 30, No. 2, pp. 410-420, Feb. 2013.

[15] M. Eguchi and Y. Tsuji, "Design of single-polarization elliptical-hole core circular-hole holey fibers with zero dispersion at $1.55 \mu \mathrm{m}$," J. Opt. Soc. Am. B, Vol. 25, No. 10, pp. 1690-1701, Oct. 2008.

[16] Z. Zhang, Y. Tsuji, and M. Eguchi, "Design of polarization splitter with single-polarized elliptical-hole core circular-hole holey fibers," IEEE Photon. Technol. Lett., Vol. 26, No. 6, pp. 541-543, Mar. 2014.

[17] Z. Zhang, Y. Tsuji, and M. Eguchi, "Study on crosstalk-free polarization splitter with elliptical-hole core circular-hole holey fibers," J. Lightw. Technol., Vol. 32, No. 23, pp. 3956-3962, Dec. 2014.

[18] M. Koshiba and Y. Tsuji, "Curvilinear hybrid edge/nodal elements with triangular shape for guidedwave problems," J. Lightw. Technol., Vol. 18, No. 5, pp. 737-743, May 2000.

[19] P. J. Roberts, D. P. Williams, B. J. Mangan, H. Sabert, F. Couny, W. J. Wadsworth, T. A. Birks, J. C. Knight and P. St. J. Russell. "Realizing low loss air core photonic crystal fibers by exploiting an antiresonant core surround," Opt. Express, Vol. 13, No. 20, pp. 8277-8285, Oct. 2005.

[20] E. N. Fokoua, F. Poletti and D. J. Richardson. "Analysis of light scattering from surface roughness in hollow-core photonic bandgap fibers," Opt. Express, Vol. 20, No. 19, pp. 20980-20989, Sep. 2012.

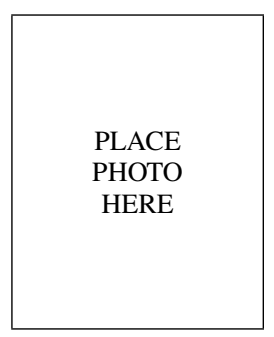

Kazuki Ichikawa ( $\left(S^{\prime}, 15\right)$ was born in Masike, Japan, on June 14, 1991. He received the B.S. degree in department of information and electrical engineering from Muroran Institute of technology, Muroran, Japan, in 2013.

Mr.Ichikawa is a student member of the Institute of Electronics, Information and Communication Engineers (IEICE), and a student member of the IEEE.

PLACE

PHOTO

HERE
Zejun Zhang ( $S^{\prime}$ 14) was born in Jiaozuo, Henan, China, on April 3, 1989. He received the B.S. degree in department of electrical engineering from Xuchang University, Xuchang, Henan, China, in 2011, and he received the M.S. degree in division of information and electronic engineering from Muroran Institute of Technology, Muroran, Japan, in 2014

Mr. Zhang is a student member of the Institute of Electronics, Information and Communication Engineers (IEICE), and a student member of the IEEE. 


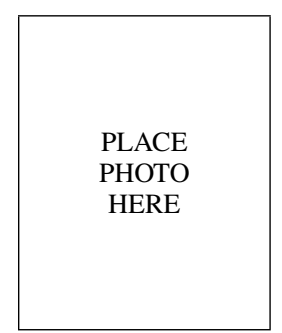

Yasuhide Tsuji (M' 97) was born in Takikawa, Japan, on December 31, 1967. He received the B.S., M.S., and Ph.D. degrees in electronic engineering from Hokkaido University, Sapporo, Japan, in 1991, 1993, and 1996, respectively.

In 1996, he joined the Department of Applied Electronic Engineering, Hokkaido Institute of Technology, Sapporo, Japan. From 1997 to 2004, he was an Associate Professor of Electronics and Information Engineering at Hokkaido University. From 2004 to 2011, he was an Associate Professor of Electrical and Electronic Engineering at Kitami Institute of Technology, Kitami, Japan. Since 2011, he has been a Professor of Division of Information and Electronic Engineering at Muroran Institute of Technology, Muroran, Japan. He has been engaged in research on optical wave electronics.

Dr. Tsuji is a member of the Institute of Electronics, Information and Communication Engineers (IEICE), the Japan Society of Applied Physics, the Optical Society of America (OSA), and IEEE. In 1997 and 1999, he was awarded the Excellent Paper Award from IEICE. In 2000, he has received the Third Millennium Medal from IEEE.

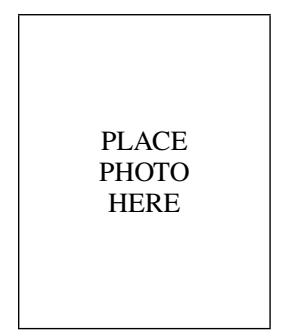

Masashi Eguchi (M'93) was born in Sapporo, Japan, on August 9, 1962. He received the B.S. degree in electronic engineering from Kitami Institute of Technology, Kitami, in 1985 and the M.S. and $\mathrm{Ph} . \mathrm{D}$ degrees in electronic engineering from Hokkaido University, Sapporo, Japan, in 1987, 1991, respectively. He joined Sony Co., Ltd., in 1987. From 1991 to 1995, he was with the Department of Industrial Design, Sapporo School of the Arts, Sapporo, Japan. And from 1995 to 1998, he was an Associate Professor of Center for Multimedia Aided Education at Muroran Institute of Technology, Muroran, Japan. Since 1998, he has been an Associate Professor of the Faculty of Photonics Science, Chitose Institute of Science and Technology, Chitose, Japan. He has been engaged in research on various optical fibers, dielectric waveguides, optical solitons, and applications of finite element method and other electromagnetic wave analysis methods.

Dr.Eguchi is a Senior Member of the Optical Society of America (OSA) and a Member of the Institute of Electronics, Information and Communication Engineers (IEICE) and the Institute of Electrical and Electronic Engineers (IEEE). 